\title{
EL ÍNDICE DE DESARROLLO HUMANO COMO INDICADOR SOCIAL
}

\author{
Raúl Enrique Molina Salazar \\ Universidad Autónoma de Madrid \\ José María Joaquín Pascual García \\ Universidad Autónoma de Madrid
}

http://dx.doi.org/10.5209/rev_NOMA.2014.v44.n4.49298

\begin{abstract}
RESUMEN.- En este trabajo se hace un análisis de la evolución del IDH como indicador social. Primero se realiza una discusión sobre la importancia de colocar en la agenda social al IDH. Posteriormente se presentan los elementos que componente el IDH. Por último se analizan los indicadores del IDH tanto a nivel global como para México.
\end{abstract}

La desigualdad existe desde los primeros hombres, esta se debe medir y se debería de crear un consenso sobre la forma de medirla y de compararla entre los distintos países, estados y entidades de distinta índole. Al hablar de la igualdad, se debe de tomar en cuenta desde qué "ángulo" estamos viendo la situación, desde la perspectiva del utilitarismo o no. También influye el ámbito de estudio de las igualdades, por ejemplo, la igualdad en salud implica una serie de igualdades asociadas como son la igualdad de inclusión, de asistencia, de educación, de libertad, etc. Amartya Sen preguntó "igualdad ¿de qué?" pues en no pocas ocasiones al pedir la igualdad respecto a " $A$ " se perjudica a otro tipo de igualdad respecto a "B".

El Índice de Desarrollo Humano(IDH), es un índice compuesto del índice de salud, índice de educación y el índice de ingresos.

Palabras clave: IDH (Indice de Desarrollo Humano), igualdad, equidad, inequidad, salud.

ABSTRACT.- This paper is an analysis of the evolution of HDI as a social indicator. A discussion on the importance of placing the social agenda to the HDI is first performed. Subsequently arise the HDI elements. Last discussed indicators of HDI both globally and for Mexico.

Inequality has existed since the first men, this should be measured and should build consensus on how to measure and compare across countries, states and entities of various kinds. Speaking of equality, it must be taken into account from what "angle" we are seeing the situation from the perspective of utilitarianism or not. Also influences the field of study of the similarities, for example, equality in health implies a series of equalities are associated as equal inclusion, support, education, freedom, etc. Amartya Sen asked "equality of what?" Because on many occasions to ask for equality with "A" harms other equality with "B".

The Human Development Index (HDI) is a composite index of the health index, education index and the income index.

\section{INTRODUCCIÓN}

La desigualdad perdura entre la sociedad desde que el ser humano vio la luz. Siempre han existido grupos vulnerables en la sociedad que, por voluntad propia o contra su voluntad, han sufrido las inclemencias de la pobreza y de la marginación.

El gran problema es ponerse de acuerdo en qué tipo de estadísticas sociales aplicar para delimitar la gravedad del asunto y disponer de suficientes elementos para atacar este mal que infecta cada vez más a la sociedad y no permite que se dé el 
suficiente crecimiento económico para establecer, de forma continuada, el Estado de Bienestar y permitir al ser humano desarrollar sus capacidades y funcionamientos, de acuerdo con las definiciones dadas por el IDH y por el propio Amartya Sen, premio nobel de economía.

Los agentes sociales, frecuentemente, por no decir siempre, constatan en su vida diaria que las estadísticas brindadas por los distintos gobiernos no se corresponden con la vivencia particular que tienen en sus vidas. Las estadísticas no son perfectas, pero son una herramienta que pueden indicarnos, mediante comparaciones interanuales, si vamos progresando, o por el contrario, vamos perdiendo terreno frente a la pobreza, la desigualdad y la marginación, traduciéndose esto por una pérdida en el bienestar, la calidad de vida, el crecimiento económico y social y desde luego en lo que respecta al desarrollo humano.

Al hablar de desigualdad, nos dice Amartya Sen, que debemos ser explícitos y delimitar nuestra preocupación. "Igualdad de qué" son las palabras exactas de Sen y sostiene que todos los enfoques referentes a la ética de las condiciones sociales, a través de los tiempos, es desear igualdad de algo. Al preocuparse por la igualdad de una variable, dice el autor, implica que la teoría en cuestión sea no igualitaria para otra variable, pues es posible que estas distintas perspectivas se encuentren en conflicto (Sen, A., 1992).

Para Sen el buscar la igualdad, tomada como actividad social central (También llamada por Sen "variable focal" siendo la variable en la que se centra el análisis: ingresos, riqueza, felicidad, libertad, oportunidades, derechos, etc.) implica aceptar la desigualdad en las periferias más remotas y nos explica algunos ejemplos como es que quien busca la igualdad de derechos a una clase de títulos no puede, de acuerdo con esto pedir igualdad de rentas; un utilitarista que pidiese igual ponderación para cada unidad de utilidad no puede, según Sen, pedir, de acuerdo con sus exigencias, igualdad de libertades o de derechos e incluso llega a decir que tampoco podría exigir que se igualen los niveles totales de utilidad de las distintas personas (Sen, A., 1992). No se debe confundir la utilidad individual con la utilidad total del conjunto de las personas o del grupo de que se hable.

Las variables son representaciones de los distintos espacios que envuelven la vida humana tales como ingresos, felicidad, salud, etc. Pero las escalas de estas variables pueden no coincidir y esto daría que no sean directamente proporcionales y que el aumento de una variable nos lleve a desequilibrios en otra. Según Sen la igualdad de oportunidades nos puede llevar a un desequilibrio en los ingresos y que estos sean muy desiguales (Sen, A., 1986).

Pero, no obstante de tener limitaciones y restricciones para la búsqueda de igualdades, Sen acepta que puede haber variables focales elegidas donde se pueden reunir una combinación de ellas afines entre sí (Sen, A., 1998), es decir, puede tener una pluralidad interna como es el caso, por ejemplo de la igualdad en salud, en donde no sólo se ve la igualdad de derecho a la salud, sino también la igualdad de inclusión, de asistencia, de trato, de educación, de libertad, etc. Aquí también se presentan igualdades confusas y complejas de acuerdo a la complejidad humana. Las variables se encuentran en espacios concretos, unas más cerca y otras más alejadas de las demás, forman grupos con similitudes que las hacen 
compatibles.

Se puede hablar de campos de aplicación donde las peticiones de igualdad sean compatibles entre sí y congruentes con el significado de dicho campo. Estos campos o espacios, nos dictarían normas específicas para lograr un bienestar aceptable para la sociedad.

La salud sería un campo de aplicación y para atender las variables que pueden desarrollarse en este espacio se debe analizar la compatibilidad entre ellas, pues la igualdad de oportunidades nos puede llevar a ingresos muy desiguales, igualdad en ingresos nos puede llevar a desigualdad de riqueza, incluso teniendo igualdad en riquezas se puede dar una desigualdad muy amplia de felicidad, en esto Sen apela a la diversidad humana pues es precisamente esta diversidad, estas diferencias la que nos plantea igualdad ¿de qué?. Cada persona necesita un tipo de igualdad ajustada a su esencia y a sus necesidades propias. Además, en no pocas ocasiones, al solicitar un tipo de igualdad "A" se va contra otro tipo de igualdad "B", por lo que se debe de delimitar éste tipo de acciones.

La diversidad humana hace que al buscar una igualdad debamos delimitarla en un espacio donde interactuará con otras variables y será antagónica de otras, una de las razones por las que el ser humano busca igualdades es la realización propia, la autorrealización y para que esto se pueda dar debe de tener a su alcance la libertad de realizarse, la libertad de elección, pues difícilmente se valorará la igualdad y la desigualdad desde un ámbito de falta de libertad para distinguir el valor de las cosas, tangibles o intangibles.

La libertad y la justicia son claves importantes para el bienestar humano, pero antes de aspirar a esto se deben tener cubiertas las necesidades conducentes a poder aspirar a un estado completo de bienestar, o por lo menos lo más completo que se pueda conforme a las tecnologías, innovaciones, educación, ambientes, físico y mental.

Al solicitar igualdad se debe tener las suficientes bases para efectuar una comparación de variables entre distintas personas o grupos. Pero el hacer comparaciones interpersonales de bienestar, se necesitan entender las bases de dichas comparaciones pues, dice Rawls, que en justicia social se deben encontrar bases objetivas con las que realizar las comparaciones y que sean bases aceptables y reconocibles por las personas (Rawls, J., 1973). Pero no se debe olvidad la complejidad existentes en cualquier relación humana, pues no sólo influyen la justicia y la libertad, sino que también tiene una gran influencia las costumbres sociales, la ética y la moral.

Para Rawls los principios de la justicia se basan en la Razón, para este autor, los hombres viven en sociedad y mediante una reflexión racional deben decidir lo que es justo o injusto. La posición original de igualdad se apoya en la teoría tradicional del contrato social viendo en esta situación hipotética una vía hacia cierta concepción de la justicia. En estas circunstancias sociales no se darían ventajas ni desventajas a nadie al escoger los principios de justicia lo que daría, dice el autor, una situación inicial equitativa. La justicia con imparcialidad comenzaría con una elección de principios (Rawls, J., 1995). 
Para Sen (Sen, A., 1998a) el camino para afrontar los retos de construir una sociedad justa es la razón, pero nos dice que es una razón objetiva, es decir que se debe buscar la objetividad con el uso de la razón y la función de razonamiento público es crucial para la búsqueda de justicia social. Rawls dice que la justicia se debe considerar desde las exigencias de equidad y ésta en términos de igualar los bienes primarios (Rawls, J., 1995).

Las personas realizan aquellas funciones que piensan tienen valor y la capacidad de estas personas para realizarlas, nos dan, dice Sen, una valoración de las condiciones sociales y mediante esto se puede valorar y tener una perspectiva más amplia sobre la igualdad y su antónimo la desigualdad (Sen, A., 1993). Distingue Sen entre funcionamientos elementales y funcionamientos más complejos y refinados, los primeros serian la alimentación, evitar las enfermedades y la muerte prematura mientras que entre los más complejos y refinados estarían el respetarse a uno mismo, el formar parte de la comunidad, etc.

\section{EL INDICE DE DESARROLLO HUMANO}

"La verdadera riqueza de una nación está en su gente", así comienza el primer capítulo del Informe de Desarrollo Humano de 1990 y sigue "El objetivo básico del desarrollo es crear un ambiente propicio para que los seres humanos disfruten de una vida prolongada, saludable y creativa" (Informe del Desarrollo Humano, 1990).

La acumulación, el mercado y todas las transacciones financieras y económicas deben de seguir un fin que según Sen y el Programa de las Naciones Unidas para el Desarrollo (PNUD) es el propio ser humano y sus necesidades, es su bienestar tanto físico, emocional o de cualquier otro orden.

Aristóteles ya apuntaba que las sociedades no se deben juzgar por sus ingresos o riquezas, pues esto se desea para obtener otros beneficios y alcanzar otras metas y objetivos. La riqueza no es el bien que se busca, sino un medio para llegar a los verdaderos bienes deseados (Informe del Desarrollo Humano, 1990).

Esta preocupación se dio también entre los pioneros de la teoría de la cuantificación económica: William Petty, Gregory King, François Quesnay, Antoine Lavoisier y Joseph Lagrange. También es evidente en los economistas políticos como son Adam Smith, David Ricardo, Robert Maltus, Karl Marx y John Stuart Mill (Informe del Desarrollo Humano, 1990).

La experiencia ha hecho palpable que en el desarrollo se debe incluir el factor social y que se debe prestar una especial atención al vínculo existente entre el crecimiento económico y el desarrollo humano. El rápido crecimiento en el PIB no necesariamente significa la reducción de carencias socioeconómicas en importantes segmentos de población de los países, ni evita problemas tales como las drogas, alcoholismo, carencias en viviendas, violencia o ruptura y destrucción de relaciones familiares (Informe del Desarrollo Humano, 1992).

Programa de las Naciones Unidas para el Desarrollo (PNUD) brinda una definición del Desarrollo Humano: 
"El desarrollo humano es un proceso mediante el cual se amplían las oportunidades del ser humano. En principio, estas oportunidades pueden ser infinitas y cambiar con el tiempo. Sin embargo, a todos los niveles del desarrollo, las tres más esenciales son disfrutar de una vida prolongada y saludable, adquirir conocimientos y tener acceso a los recursos necesarios para lograr un nivel de vida digno. Si no se poseen estas oportunidades esenciales, muchas otras alternativas continuarán siendo inaccesibles" (Informe del Desarrollo Humano, 1992).

"El desarrollo humano tiene dos aspectos. La formación de capacidades humanas tales como un mejor estado de salud, conocimientos y destrezas - y el uso que la gente hace de las capacidades adquiridas - para el descanso, la producción o las actividades culturales, sociales y políticas. Si el desarrollo humano no consigue equilibrar estos dos aspectos, puede generarse una considerable frustración humana" (Informe del Desarrollo Humano, 1990).

La carencia de oportunidades de acceso a los servicios de salud puede ser traducida como una inequidad en lo referente a la salud de los grupos excluidos y discriminados siendo que la Organización Mundial de la Salud entiende por inequidades las desigualdades evitables en materia de salud entre grupos de población en un mismo país o entre países. Las condiciones sociales y económicas y los efectos que esto produce en la vida de la población determina el riesgo de enfermarse y las medidas que se toman para evitarlo ${ }^{1}$.

Las desigualdades entre países están muy bien documentadas, por ejemplo en los países con muy alto desarrollo humano se tiene una esperanza de vida de 80 años mientras que en los de bajo desarrollo humano sólo alcanza los 58.7 años. La escolaridad promedio va desde los 4.2 años hasta los 11.3, el PIB va desde los US\$1,585 hasta los US\$33,352 per cápita (Tabla 5)

La sociedad actual vive en un estado de "gradientes sociales" donde los más pobres entre los pobres son también los que tienen peor salud. Dentro de los países también se dan situaciones anómalas e inequidades. La OMS nos da ciertos ejemplos muy significativos ${ }^{2}$ :

Ejemplos de inequidades sanitarias dentro de un mismo país:

- $\quad$ En Bolivia, la tasa de mortalidad infantil de los bebés de madres que no han cursado estudios supera los 100 por 1000 nacidos vivos, mientras que la de los bebés de madres que han cursado por lo menos estudios secundarios es inferior a 40 por 1000 nacidos vivos;

La esperanza de vida de los aborígenes australianos es considerablemente inferior (59,4 para los varones y 64,8 para las mujeres) que la de los australianos no aborígenes (76,6 y 82,0 , respectivamente);

\footnotetext{
${ }^{1}$ Organización Mundial de la Salud (2012)

${ }^{2}$ Organización Mundial de la Salud (2012)
} 
La esperanza de vida al nacer de los varones en el barrio de Calton, Glasgow, es de 54 años, 28 años menor que la de los varones de Lenzie, a unos pocos kilómetros de distancia;

\section{La prevalencia de discapacidades de larga duración entre los varones} europeos de 80 años o más es del 58,8\% para los poco instruidos, y del 40,2\% para los más instruidos.

Por último, Amartya Sen señala que en la conceptualización de la pobreza se debe de tomar en cuenta, que es una característica de los pobres como grupo social ${ }^{3}$. Sen señaló el concepto de pobreza a partir de las capacidades, esto es lo que la gente puede hacer y ser y define a la pobreza como la ausencia de capacidades básicas que le permiten a cualquier persona insertarse en la sociedad ${ }^{4}$. Dice Sen que la pobreza no es cuestión de escaso bienestar, ni de falta de riqueza o ingreso, sino de capacidades básicas ${ }^{5}$ y de carencias que impidan a una persona llevar una vida digna por encima de una línea imaginaria por debajo de la cual se estaría en situación de pobreza, moderada o extrema, dependiendo de lo profundo que se encuentre la línea divisoria entre los grupos o las personas.

\section{NACIMIENTO Y COMPONENTES DEL IDH: EDUCACIÓN Y SALUD}

Construir un modelo es siempre una tarea compleja. Resulta imposible el incluir todas las variables en un modelo, por lo que resulta básico el poder elegir las variables adecuadas para reflejar la realidad en forma precisa.

El IDH nació en 1990 por la preocupación de medir el desarrollo humano. El Programa de las Naciones Unidas para el Desarrollo (PNUD), en su informe de 1990 declara que lo ideal sería incluir muchas variables pero que el exceso de indicadores podría dar una imagen confusa y ser motivo de desvío para los diseñadores de políticas públicas sugiriendo la medición del desarrollo humano centrándose en tres elementos básicos de la vida humana, a saber, la longevidad, los conocimientos y los niveles de vidadignos (Informe del Desarrollo Humano, 1990).

Para el elemento de longevidad se tomó en consideración el indicador de la esperanza de vida. Se tomó esto en cuenta pues ya de por sí, la esperanza de vivir es muy importante y este dato lleva consigo una estrecha relación con la salud y la nutrición, entre otros indicadores (Informe del Desarrollo Humano, 1990).

El segundo componente es la alfabetización ya que el saber leer y escribir es básico para iniciar la educación en las personas y así llevar una vida productiva siendo este dato esencial en cualquier medición de desarrollo humano (Informe del Desarrollo Humano, 1990).

El tercer componente se refiere al manejo de los recursos para llevar un nivel de vida digno, según el Programa de las Naciones Unidas para el Desarrollo (PNUD) es

\footnotetext{
${ }^{3}$ Sen, A. (1986)

${ }^{4}$ Sen, A. (1992)

5 Sen, A. (1993)
} 
quizás el más difícil de medir en forma sencilla pues necesita datos sobre el acceso a la tierra, el crédito, el ingreso y otros recursos. El ingreso se forma a partir de las cifras reales de PIB ajustadas al poder adquisitivo pues son las cifras que proporcionan mejores aproximaciones del poder de compra y de lograr un mejor control sobre los recursos necesarios para alcanzar un nivel de vida adecuado (Informe del Desarrollo Humano, 1990).

Según el Programa de las Naciones Unidas para el Desarrollo (PNUD) existen varias formas de calcular el PNB y muestra en su informe (1990) cuatro países (Tabla 1): Panamá, Brasil, Malasia y Costa rica siendo Panamá quien ostenta mayor ingreso per cápita, seguido de Brasil, de Malasia y por último de Costa Rica. Al tomar el PIB real según el poder adquisitivo, cambia el orden de los países: Brasil, Panamá, Malasia y Costa Rica. Utilizando el coeficiente de Gini para ajustar la distribución, resulta que la clasificación original se invierte totalmente quedando, en orden: Costa Rica, Malasia, Brasil y Panamá. Las correcciones de distribución se realizan igualmente para la esperanza de vida y cualquier otro indicador y revelan una diferencia significativa en las evaluaciones del desempeño de un país (Informe del Desarrollo Humano, 1990).

TABLA 1: DIFERENCIA ENTRE PIB, PIB AJUSTADO AL PODER ADQUISITIVO Y PIB AJUSTADO A LA DISTRIBUCIÓN.

\begin{tabular}{|l|l|l|l|l|}
\hline PAIS & $\begin{array}{l}\text { PIB } \\
\text { Per cápita } \\
\text { (US\$) } \\
1987\end{array}$ & $\begin{array}{l}\text { PIB real } \\
\text { Per cápita } \\
\text { (ppa } \$) \\
1987\end{array}$ & $\begin{array}{l}\text { Coeficiente } \\
\text { Gini de } \\
\text { Desigualdad }\end{array}$ & $\begin{array}{l}\text { PIB per cápita } \\
\text { Ajustado a la } \\
\text { distribución } \\
\text { (ppa\$) }\end{array}$ \\
\hline Panamá & $2,240(1)$ & $4,010(2)$ & 0.57 & $1,724(4)$ \\
\hline Brasil & $2,020(2)$ & $4,310(1)$ & 0.57 & $1,852(3)$ \\
\hline Malasia & $1,810(3)$ & $3,850(3)$ & 0.48 & $2,001(2)$ \\
\hline Costa Rica & $1,610(4)$ & $3,760(4)$ & 0.42 & $2,180(1)$ \\
\hline
\end{tabular}

Fuente: Informe del Desarrollo Humano de 1990.

Para crear el índice se tomaron los valores mínimos (la serie máxima de privación igual a uno) y los valores deseables o adecuados (ningún rango de privación igual a cero). Se tomaron, en 1990, los valores más bajos de 1987, dando como resultado el valor mínimo de esperanza de vida de 42 años (Afganistán, Etiopía y Sierra Leona), de alfabetismo el $12 \%$ (Somalia) y para el PIB per cápita ajustado al poder adquisitivo se tomó US\$220 (Zaire). Los valores de logros deseables o adecuados fueron los de Japón en 1987 con una esperanza de vida de 78 años, una tasa de alfabetización del 100\% y un ingreso promedio de la "línea de pobreza" en 9 países industrializados, ajustados según las paridades del poder adquisitivo de US\$4,861 (Australia, Canadá, República Federal Alemana, Holanda, Noruega, Suecia, Suiza, Reino Unido y los Estados Unidos) (Informe del Desarrollo Humano, 1990).

TABLA 2: VALORES MÍNIMOS Y VALORES DESEABLES DEL IDH

\begin{tabular}{|l|l|l|}
\hline & Valores mínimos & $\begin{array}{l}\text { Valores deseables } \\
\text { adecuados }\end{array}$ \\
\hline Esperanza de vida & 42 años & 78 años \\
\hline Alfabetismo & $12 \%$ & $100 \%$ \\
\hline PIB per cápita ajustado & US\$220 & US $\$ 4,861$ \\
\hline
\end{tabular}

Fuente: Informe del desarrollo humano de 1990 
Los valores mínimos y deseables o adecuados forman una escala de uno a cero para cada medida de privación. Al situar un país en un punto de cada escala y promediar las tres escalas se obtiene un índice promedio de privación humana, el cual restándolo de 1 nos indica el índice de desarrollo humano (Informe del Desarrollo Humano, 1990).

\section{IDH EN EL MUNDO}

El Programade las Naciones Unidas para el Desarrollo (PNUD) añadió tres nuevos indicadores a la familia de mediciones del Índice de Desarrollo Humano: el índice de desarrollo humano ajustado por la desigualdad, el índice de desigualdad de género y el índice de pobreza multidimensional, haciendo énfasis en que la desigualdad y la pobreza ocupan un lugar central en el desarrollo humano (Informe del Desarrollo Humano, 2010).

Los datos del informe 2011 sobre desarrollo humano se dividen en (Tabla 3):

1- Países con desarrollo humano muy alto (DHMA) ${ }^{6}$

2- Países con desarrollo humano alto $(\mathrm{DHA})^{7}$

3- Países con desarrollo humano medio (DHM) ${ }^{8}$

4- Países con desarrollo humano bajo $(\mathrm{DHB})^{9}$

TABLA 3: VALORES DE COMPONENTES DEL IDH AGRUPADOS POR PAÍSES SEGÚN EL VALOR DE SU DH.

\begin{tabular}{|l|l|l|l|l|l|l|}
\hline Agrupación & IDH & $\begin{array}{l}\text { Esperanza } \\
\text { de vida al } \\
\text { nacer }\end{array}$ & $\begin{array}{l}\text { Años } \\
\text { promedio } \\
\text { de } \\
\text { escolaridad }\end{array}$ & $\begin{array}{l}\text { Esperados de } \\
\text { escolarización }\end{array}$ & $\begin{array}{l}\text { PIB } \\
\text { per } \\
\text { cápita }\end{array}$ & $\begin{array}{l}\text { IDH no } \\
\text { referidos } \\
\text { a } \\
\text { ingresos }\end{array}$ \\
\hline DHMA & 0.889 & 80 & 11.3 & 15.9 & 33,352 & 0.918 \\
\hline DHA & 0.741 & 73.1 & 8.5 & 13.6 & 11,579 & 0.769 \\
\hline DHM & 0.630 & 69.7 & 6.3 & 11.2 & 5,276 & 0.658 \\
\hline
\end{tabular}

\footnotetext{
${ }^{6}$ En orden decreciente: Noruega, Australia, Países Bajos, Estados Unidos, Nueva Zelandia, Canadá, Irlanda, Liechtenstein, Alemania, Suecia, Suiza, Japón, Hong Kong, China (RAE), Islandia, República de Corea, Dinamarca, Israel, Bélgica, Austria, Francia, Eslovenia, Finlandia, España, Italia, Luxemburgo, Singapur, República Checa, Reino Unido, Grecia, Emiratos Arabes Unidos, Chipre, Andorra, Brunei Darussalam, Estonia, Eslovaquia, Malta, Qatar, Hungría, Polonia, Lituania, Portugal, Bahrein, Letonia, Chile, Argentina, Croacia y Barbados.

${ }^{7}$ En orden decreciente: Uruguay, Palau, Rumania, Cuba, Seychelles, Bahamas, Montenegro, Bulgaria, Arabia Saudita, México, Panamá, Serbia, Antigua y Barbuda, Malasia, Trinidad y Tobago, Kuwait, Libia, Belarús, Federación de Rusia, Granada, Kazajstán, Costa Rica, Albania, Líbano, Saint Kitts y Nevis, Venezuela (República Bolivariana de), Bosnia y Herzegovina, Georgia, Ucrania, Mauricio, Ex República Yugoslava de Macedonia, Jamaica, Perú, Dominica, Santa Lucía, Ecuador, Brasil, San Vicente y las Granadinas, Armenia, Colombia, Irán (República Islámica de), Omán, Tonga, Azerbaiyán, Turquía, Belice y Tunez.

${ }^{8}$ En orden decreciente: Jordania, Argelia, Sri Lanka, República Dominicana, Samoa, Fiji, China, Turkmenistán, Tailandia, Suriname, El Salvador, Gabón, Paraguay, Bolivia (Estado Plurnacional de), Maldivas, Mongolia, Moldova (república de), Filipinas, Egipto, Territorios Palestinos Ocupados, Uzbekistán, Micronesia (Estados Federados de), Guyana, Botswana, República Árabe Siria, Namibia, Honduras, Kiribati, Sudáfrica, Indonesia, Vanuatu, Kirguistán, Viet Nam, Nicaragua, Marruecos, Guatemala, Iraq, Cabo Verde, India, Ghana, Guinea Ecuatorial, Congo, República Democrática de Lao, Camboya, Swazilandia y Buthán.

${ }^{9}$ En orden decreciente: Islas Salomón, Kenya, Santo Tomé y Principe, Pakistán, Bangladesh, Timor-Leste, Angola, Myanmar, Camerún, Madagascar, Tanzania (República Unida de), Papua Nueva Guinea, Yemen, Senegal, Nigeria, Nepal, Haití, Mauritania, Lesotho, Uganda, Togo, Comoras, Zambia, Djibouti, Rwanda, Benin, Gambia, Sudán, Côte d' Ivoire, Malawi, Afganistán, Zimbabwe, Etiopía, Malí, Guinea Bissau, Eritrea, Guinea y República Centroafricana.
} 


\begin{tabular}{|l|l|l|l|l|l|l|}
\hline DHB & 0.456 & 58.7 & 4.2 & 8.3 & 1,585 & 0.478 \\
\hline MUNDIAL & $\mathbf{0 . 6 8 2}$ & $\mathbf{6 9 . 8}$ & $\mathbf{7 . 4}$ & $\mathbf{1 1 . 3}$ & $\mathbf{1 0 , 0 8 2}$ & $\mathbf{0 . 6 8 3}$ \\
\hline
\end{tabular}

Datos del Anexo del Informe de Desarrollo Humano $2011^{10}$

Se ha comprobado que el desarrollo humano va unido a la esperanza de vida, a la educación y a los ingresos habiendo una enorme diferencia entre los países con muy alto desarrollo humano y los calificados como bajo desarrollo humano, siendo que estos tienen un $26.63 \%$ menos años de vida, un $62.83 \%$ menos en promedio de escolaridad y un ingreso inferior en $95.24 \%$. Esto da idea de la marginación a la que están expuestos los integrantes de estos grupos de bajo desarrollo humano.

\section{IDH EN MEXICO}

México forma parte de la OCDE desde el 18 de mayo de 1994 y la situación de desigualdad de México con respecto a los demás países de la OCDE es la siguiente:

1) Tiene el mayor número de mortalidad infantil ${ }^{11}$

2) Tiene el menor gasto público social en salud ${ }^{12}$

3) Tiene el menor gasto total en salud per cápita ${ }^{13}$

A nivel mundial el gasto público total en salud per cápita tiene un rango de US $\$ 8,350$ siendo el mayor de US\$8.362 (EUA) y el menor US\$12 (Eritrea), estando México con US\$604, equivaliendo ésto a decir que el mayor gasto es 696.83 veces mayor que el menor y 13.84 veces mayor que el de México. Esto equivale a decir que lo que gastan los EUA en una sola persona es equivalente a lo que gasta México para 14 personas y lo que gasta Eritrea para 697 personas. En lo que se refiere al porcentaje del PIB dedicado al gasto público en salud, los datos de que se disponen arrojan una diferencia de 16.1 del PIB entre el mayor (EUA: $17.9 \%$ ) y el menor (Qatar $1.8 \%$ ) y de 11.6 con respecto México, el $6.3 \%$. Siendo en números relativos casi 10 veces más en EUA que en Qatar y casi tres veces más que en México. La

\footnotetext{
10 - Índice de Desarrollo Humano (IDH): Índice compuesto que mide el promedio de lso avances en tres dimensiones básicas del desarrollo humano: vida larga y saludable, conocimientos y nivel de vida digno.

- Esperanza de vida al nacer: años que vivirá un recién nacido si los patrones de mortalidad por edades imperantes en el momento de su nacimiento siguieran siendo los mismos a lo largo de su vida.

- Años promedio de escolaridad: años promedio de escolaridad que reciben las personas de 25 años y más, según los niveles de logros educacionales de la población de acuerdo con la duración de cada nivel.

- Años esperados de escolarización: años de instrucción que un menor en edad de ingresar a la escuela puede esperar recibir si los patrones vigentes de las tasas de matriculación específicas por edad se mantuvieran constantes durante toda su vida.

- INB per cápita: el ingreso total de una economía generado por su producción y la propiedad de los factores de producción, menos los ingresos pagados por el uso de los factores de producción de propiedad del resto del mundo, convertido a dólares estadounidenses usando las tasas de paridad del poder adquisitivo (PPA), dividido por la población a mitad de año.

-IDH no referido a ingresos: valor del IDH calculado sólo a partir de los indicadores de esperanza de vida y educación.

${ }^{11}$ Datos recabados en línea de: http://www.oecd-ilibrary.org/fr/social-issues-migration-health/mortaliteinfantile_20758499-table9.

${ }^{12}$ Datos recabados en línea de : http://stats.oecd.org/BrandedView.aspx?oecd_bv_id=socx-data-fr\&doi=data00166-fr.

${ }^{13} \mathrm{http} / /$ www.oecd-ilibrary.org/fr/social-issues-migration-health/data/statistiques-de-l-ocde-sur-les-depensessociales_socx-data-fr.
} 
mortalidad infantil para estos países referenciados arriba es de: para los EUA, 8 casos de cada 1000 nacimientos, en Eritrea, de 70, en Qatar de 8 y en México de 17. En el Mundo, los países con muy alto desarrollo humano tienen 16.41 casos de incidencia de la tuberculosis, es decir por cada 100,000 habitantes (promedio de casos: 2,377 ), mientras que en los países de bajo desarrollo humano la incidencia representa 266.54 casos por 100,000 habitantes, (promedio de casos: 61,727) ${ }^{14}$.

Las regiones más desfavorecidas son, como es históricamente un hecho en pobreza y desigualdad, Chiapas, Oaxaca, Guerrero, Michoacán, Veracruz, etc. Regiones todas ellas con carencias destacables (Gráfica 1).

El Índice de desarrollo humano (IPC) del Programa de las Naciones Unidas para el Desarrollo (PNUD) desde el inicio priorizó la capacidad de las personas para dar alternativas elegibles de vida que se consideren valiosas (PNUD, 2012). El IDH da prioridad a la libertad de las personas y no solo a tanto a la acumulación de ingresos (PNUD, 2012).

El IDH sintetiza tres índices que son el de educación, el de salud y el de ingreso. En México el IDH estimó con la información del 2010 que México se halla ubicado con un IDH de 0.7390, Tabla

GRÁFICA 1: ÍNDICE DE DESARROLLO HUMANO POR ESTADOS, 2010

${ }^{14}$ Datos recabados del Banco Mundial en línea: http://datos.bancomundial.org/tema/salud. 
Gráfica 2. Índice de desarrollo humano, 2010

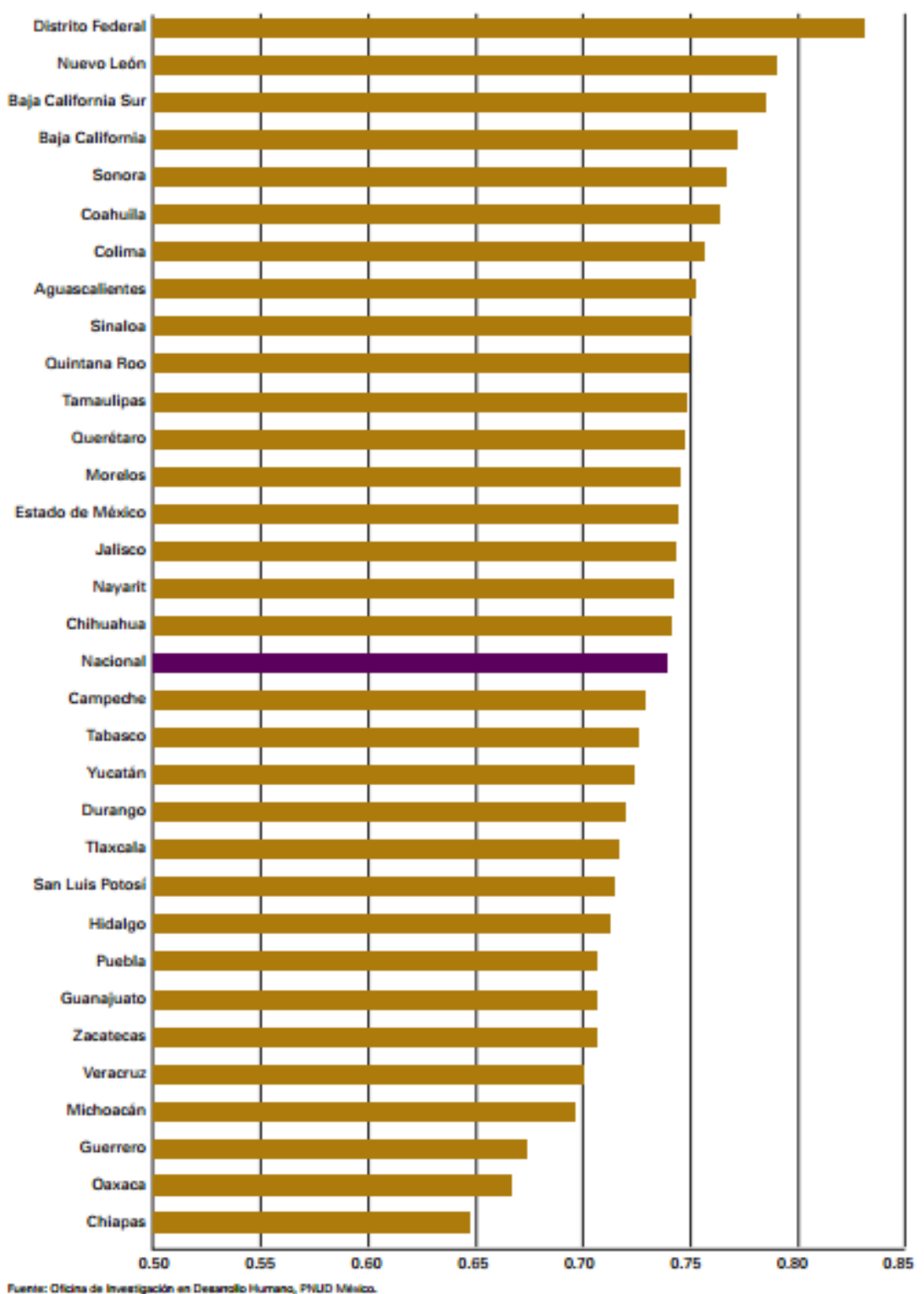

Fuente: PNUD (2012)

La gráfica 2 muestra al Distrito Federal, Nuevo león y Baja California Sur como las entidades con mayor IDH, los cuales alcanzan niveles de desarrollo similares a la República Checa, Polonia y Croacia respectivamente, mientras que Chiapas,

Oaxaca y Guerrero se encuentran al final de la tabla con un IDH comparable al de Bolivia, Argelia y Brasil (Gráfica 1). 
GRÁFICA 2: IDH DE ALGUNAS ENTIDADES FEDERATIVAS EN UN CONTEXTO INTERNACIONAL, 2010.

IDH de algunas entidades federativas en un contexto internacional, 2010

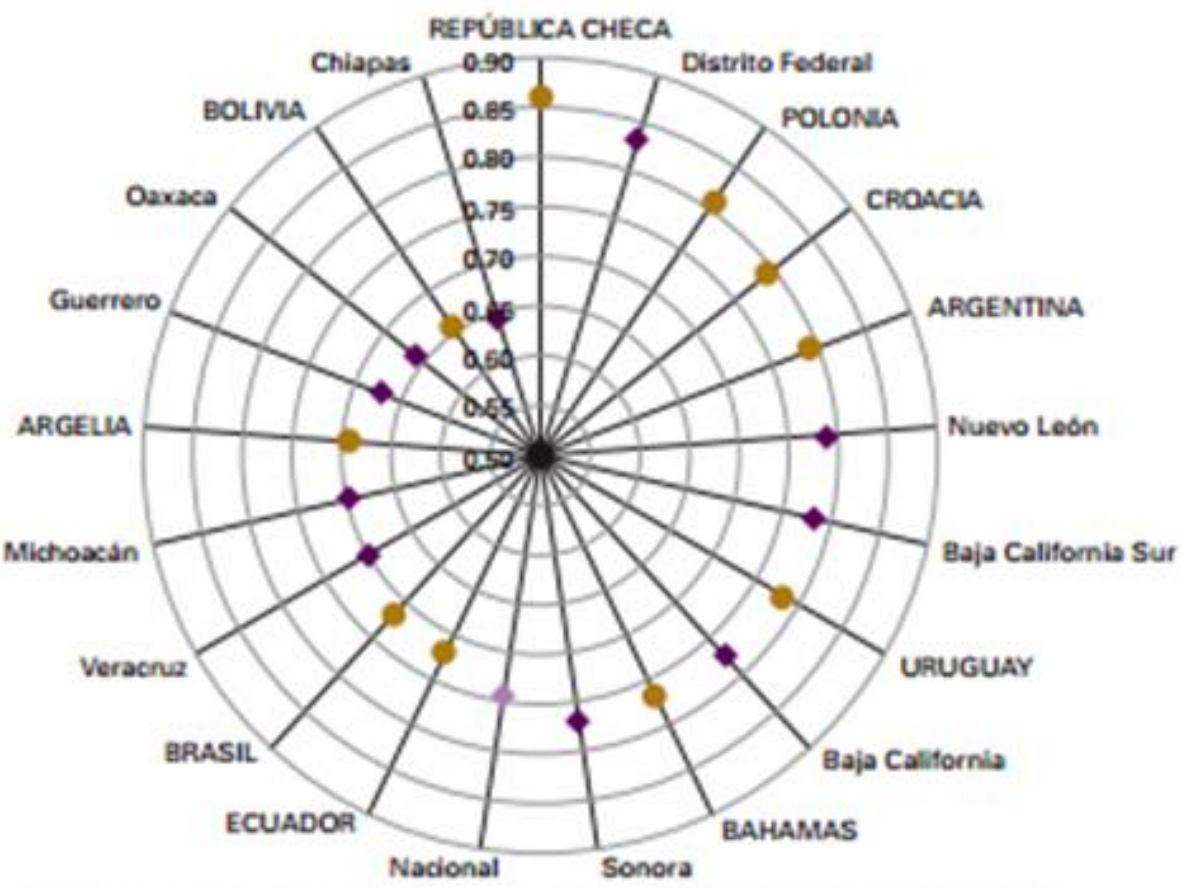

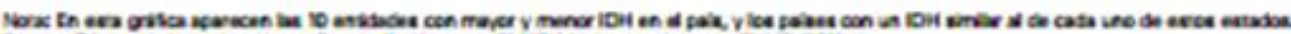

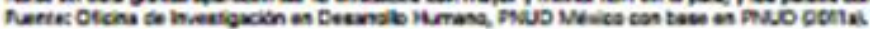

Fuente: PNUD (2012)

TABLA 4: DESGLOSE DEL IDH EN MÉXICO, 2010

\begin{tabular}{l|r|r|r|r|}
\hline \multicolumn{5}{|l|}{$\begin{array}{l}\text { Entidad Federativa Valor del índice de desarrollo } \\
\text { humano (IDH) }\end{array}$} \\
\hline Entidad & IS 2010 & IE 2010 & \multicolumn{1}{c|}{$\begin{array}{l}\text { IIng } \\
2010\end{array}$} & $\begin{array}{l}\text { IDH } \\
2010\end{array}$ \\
\hline Aguascalientes & 0.8775 & 0.6921 & 0.7006 & 0.7521 \\
\hline Baja_California & 0.8869 & 0.7073 & 0.7325 & 0.7717 \\
\hline Baja_California_Sur & 0.8867 & 0.7521 & 0.7256 & 0.7851 \\
\hline Campeche & 0.873 & 0.6562 & 0.6766 & 0.7291 \\
\hline Coahuila & 0.8688 & 0.7232 & 0.708 & 0.7634 \\
\hline Colima & 0.8799 & 0.703 & 0.7006 & 0.7567 \\
\hline Chiapas & 0.8587 & 0.5541 & 0.5688 & 0.6468 \\
\hline Chihuahua & 0.8836 & 0.6758 & 0.6791 & 0.7402 \\
\hline Distrito_Federal & 0.888 & 0.8498 & 0.7595 & 0.8307 \\
\hline Durango & 0.864 & 0.6681 & 0.6448 & 0.7193 \\
\hline Guanajuato & 0.8784 & 0.6153 & 0.6508 & 0.7059 \\
\hline Guerrero & 0.8492 & 0.5902 & 0.6092 & 0.6733 \\
\hline Hidalgo & 0.8697 & 0.657 & 0.6329 & 0.7124 \\
\hline
\end{tabular}




\begin{tabular}{|l|r|r|r|r|} 
Jalisco & 0.8774 & 0.6728 & 0.6959 & 0.7434 \\
\hline Estado_de_México & 0.8839 & 0.6841 & 0.6816 & 0.7442 \\
\hline Michoacán & 0.8696 & 0.5959 & 0.6507 & 0.6958 \\
\hline Morelos & 0.8854 & 0.6999 & 0.6671 & 0.7449 \\
\hline Nayarit & 0.8726 & 0.6872 & 0.6826 & 0.7425 \\
\hline Nuevo_León & 0.8775 & 0.7448 & 0.7543 & 0.79 \\
\hline Oaxaca & 0.8616 & 0.5679 & 0.6046 & 0.6663 \\
\hline Puebla & 0.8758 & 0.6356 & 0.6321 & 0.706 \\
\hline Querétaro & 0.8792 & 0.69 & 0.6875 & 0.7471 \\
\hline Quintana_Roo & 0.8912 & 0.6576 & 0.7163 & 0.7488 \\
\hline San_Luis_Potosí & 0.8746 & 0.6408 & 0.6505 & 0.7144 \\
\hline Sinaloa & 0.8658 & 0.7115 & 0.6859 & 0.7504 \\
\hline Sonora & 0.8755 & 0.725 & 0.7107 & 0.7669 \\
\hline Tabasco & 0.8671 & 0.6902 & 0.6394 & 0.726 \\
\hline Tamaulipas & 0.8729 & 0.6988 & 0.6848 & 0.7475 \\
\hline Tlaxcala & 0.8868 & 0.6719 & 0.6165 & 0.7162 \\
\hline Veracruz & 0.8558 & 0.6189 & 0.6467 & 0.6997 \\
\hline Yucatán & 0.8742 & 0.6474 & 0.6678 & 0.723 \\
\hline Zacatecas & 0.8828 & 0.6306 & 0.6312 & 0.7057 \\
\hline Nacional & $\mathbf{0 . 8 7 4 3}$ & $\mathbf{0 . 6 7 7 9}$ & $\mathbf{0 . 6 8 0 9}$ & $\mathbf{0 . 7 3 9}$ \\
\hline Fuente:PND
\end{tabular}

Fuente: PNUD (2012). Siendo IS: Índice de salud, IE: Índice de educación, IIng: Índice de ingreso e IDH: Índice de desarrollo humano.

De la tabla 4 se puede observar que en el Distrito Federal se encuentra un IDH superior al promedio nacional en un $12 \%$ y el de educación un $25 \%$. En contraste el IDH de Oaxaca se encuentra un $10 \%$ abajo del promedio nacional, el de educación es un $16 \%$ menor al nacional. Se puede establecer una brecha entre el índice de educación entre el Distrito Federal y Oaxaca del $41 \%$, en ingresos del 24 $\%$, en salud del $3,1 \%$ y del IDH total del $22 \%$.

En México, los 5 estados con menor desarrollo humano son: Chiapas, Oaxaca, Guerrero, Michoacán y Veracruz se encuentra un círculo de pobreza y marginación (Tabla 4). En lo estados con menor IDH se encuentran los más pobres, y son los más propensos a padecer enfermedades infecciosas y tuberculosis, homicidios, mortalidad materna, mortalidad infantil, desnutrición, anemia nutricional y tienen el menor número de partos atendidos, de gasto público en salud, de médicos y enfermeras y de población derechohabiente .

\section{IDH e INEQUIDAD EN SALUD}

En 2005 la OMS creó la Comisión sobre Determinantes sociales de la Salud como respuesta a la creciente preocupación suscitada por las inequidades persistentes y cada vez mayores en el área de la salud. Los determinantes sociales de la salud son las circunstancias en que nacen, crecen, viven, trabajan y envejecen las personas. Según la OMS estas circunstancias son resultado de la distribución del dinero, el poder, los recursos a nivel mundial, nacional y local que depende de las políticas 
$\operatorname{adoptadas}^{15}$.

Para el Dr. Cesar Gattini entre los determinantes sociales de relevancia en salud destacan el ingreso económico, empleo, pobreza, educación, condiciones de vivienda, agua y saneamiento básico, ruralidad y algunas condiciones étnicas, culturales y de migración ${ }^{16}$, dice el autor que este conjunto de factores está generalmente interrelacionado y que se concentra en grupos de población con privación de condiciones de vida saludable, vulnerables y excluidos, con menos oportunidad de acceso a los servicios de salud.

La carencia de oportunidades de acceso a los servicios de salud puede ser traducida como una inequidad en lo referente a la salud de los grupos excluidos y discriminados siendo que la Organización Mundial de la Salud entiende por inequidades a las desigualdades evitables en materia de salud entre grupos de población en un mismo país o entre países. El IDH es más bajo cuando se da la pobreza y la desigualdad en un Estado, por lo que comprueba que la pobreza y la desigualdad social impiden el desarrollo humano y el estado de bienestar.

TABLA 5.- Estimaciones de la correlacion entre el IDH, pobreza, INIQIIS, Acceso a la Seguirdad Social e ingresos.

\begin{tabular}{|c|c|c|c|c|c|c|c|}
\hline & $\begin{array}{c}\text { ÍNDICE } \\
D E \\
\text { POBREZ } \\
A\end{array}$ & $\begin{array}{c}\text { INIQUI } \\
S\end{array}$ & $\begin{array}{c}\text { Pobl. } \\
\text { Sit. } \\
\text { Pobrez } \\
\text { a }\end{array}$ & $\begin{array}{c}\text { Al } \\
\text { menos } \\
1 \\
\text { carenci } \\
a\end{array}$ & $\begin{array}{c}\text { Carenci } \\
\text { a } \\
\text { acceso } \\
\text { seg. } \\
\text { Social }\end{array}$ & $\begin{array}{c}\text { Ingreso } \\
\text { inferior a } \\
\text { línea de } \\
\text { bienesta } \\
r\end{array}$ & $\begin{array}{l}\text { IDH } \\
2010\end{array}$ \\
\hline $\begin{array}{l}\text { INDICE DE } \\
\text { POBRFZA }\end{array}$ & & & & & & & \\
\hline POBREZA & 1 & & & & & & \\
\hline $\begin{array}{l}\text { INIQUIS } \\
\text { Pobl. sit. }\end{array}$ & 0.6829 & 1 & & & & & \\
\hline Pobreza & 1.0000 & 0.6829 & 1 & & & & \\
\hline $\begin{array}{l}\text { Al menos } 1 \\
\text { carencia } \\
\text { Carencia } \\
\text { acceso Seg. }\end{array}$ & 0.9154 & 0.7418 & 0.9154 & 1 & & & \\
\hline $\begin{array}{l}\text { Social } \\
\text { Ingreso inferior } \\
\text { a línea de }\end{array}$ & 0.8349 & 0.6591 & 0.8349 & 0.9407 & 1 & & \\
\hline bienestar & 0.9845 & 0.6314 & 0.9845 & 0.8455 & 0.7696 & 1 & \\
\hline $\mathrm{IDH} 2010$ & -0.9284 & -0.6461 & -0.9284 & -0.8420 & -0.7374 & -0.9255 & 1 \\
\hline
\end{tabular}

Fuente: estimaciones propias

En la tabla de correlaciones se aprecia que el INIQUIS (desigualdad evitable en salud) explica en un $68 \%$ el índice de pobreza, la carencia de acceso a la seguridad social en un $83 \%$, la población con al menos una carencia en un $91 \%$ y el ingreso inferior a línea de bienestar en el $98 \%$. Por otra parte el IDH viene con signo negativo, lo que implica que a más pobreza, menos índice de desarrollo humano y a menos pobreza, más índice de desarrollo humano y da negativo con respecto a las carencias y al ingreso inferior a la línea de pobreza. Llama la atención la alta

\footnotetext{
${ }^{15}$ Organización Mundial de la Salud (2012)

${ }^{16}$ Gattini, C. y Ruíz, P. (2012)
} 
correlación existente entre la carencia de seguridad social y la pobreza, tanto en el índice de pobreza como en la población en situación de pobreza por el desamparo que esto provoca a la situación de salud de la población.

El Consejo Nacional de Población indica en su informe sobre los resultados del 2010 (CONAPO 2010), los datos manejados por esta institución son que a pesar de que la mayoría de la población, en México, se encuentra en un grado bajo o muy bajo de marginación, existen proporciones importantes con grado muy alto, alto y medio y esta situación pone de manifiesto la profunda desigualdad que existe en la participación del proceso de desarrollo y el disfrute de sus beneficios en la sociedad mexicana. Pero al hacer un análisis sobre las localidades, el resultado es que casi el $80 \%$ se encuentra en un grado muy alto y alto de marginación y menos del $10 \%$ se ubican en las categorías de baja y muy baja ${ }^{17}$.

Encontró CONAPO 2010 que en las localidades con muy alta marginación, el 37.6\% de las personas con más de 15 años es analfabeta, el $61.2 \%$ no terminó la primaria. Las condiciones de vivienda son sumamente precarias, sobre todo para la salud de las personas que habitan en ellas: $67.8 \%$ no cuenta con agua entubada, $31.2 \%$ no posee energía eléctrica, $46.5 \%$ tiene piso de tierra, $37.2 \%$ no tiene excusado, $87.3 \%$ no cuenta con refrigerador y el promedio de ocupantes por cuarto es de 2.3, permitiéndose hacer más ineficiente la economía familiar que con un menor índice de personas por cuarto. Estos datos nos muestran como la falta de oportunidades de educación y de salud son unos de los principales problemas que padecen los habitantes de las localidades más vulnerables.

Con todos estos datos en la mano queda patente el que una gran parte de la población se encuentra excluida de la protección social en salud, de educación, vivienda y de otros aspectos fundamentales que les permita gozar de una buena calidad de vida. La declaración de Alma Ata del 78 declara inaceptable la grave desigualdad existente en la salud de la población centrándose en la atención primaria, por lo que para Alma Ata la verdadera desigualdad en salud se basa en la desigualdad en lo que respecta a la atención en salud, siendo que la falta de atención, la inadecuada atención o una atención desigual daña gravemente el derecho que todas las personas tienen de vivir una vida gozando de la máxima salud que se les pueda brindar dependiendo de los adelantos científicos y técnicos con que se disponga en cada momento.

Si bien todas las personas tienen derecho a ser atendidas eficazmente en lo que respecta a su salud, es bien cierto que en el tiempo actual se está dando un reajuste demográfico, la estructura poblacional está cambiando: disminuye la población de menos de 15 años y aumenta la población de más de 15 años, siendo que cada día la población será de más edad y esto provocará problemas económicos importantes para que los gobiernos atiendan las demandas sociales de sus ciudadanos. Se tienen identificados cuatro mecanismos de financiamiento que son: el gasto público (impuestos), los seguros privados, los seguros nacionales de salud y el cobro a los usuarios. Todos estos mecanismos tienen impactos positivos en lograr generar ingresos, pero también tienen efectos negativos en el acceso a los servicios de salud generando carencias de oportunidades.

\footnotetext{
${ }^{17}$ CONAPO (2010)
} 


\section{CONCLUSIÓN}

Uno se pregunta ¿Por qué en los países más pobres las personas tienen, por lo general, peor estado de salud y peor calidad de vida que en los países más aventajados y más ricos? $\mathrm{O}$, incluso, yendo más lejos, podríamos cuestionarnos ¿por qué en un mismo país se dan esas enormes diferencias en la distribución y en el bienestar? Unos Estados o unos municipios están más desvinculados de la vida de bonanza que otros. Para efectuar un estudio coherente sobre la marginación, la exclusión social y por lo tanto sobre la pobreza y la desigualdad se debe bajar a nivel de los municipios, de los barrios, e incluso de las colonias.

La carencia de oportunidades de acceso a los servicios de salud puede ser traducida como una inequidad en lo referente a la salud de los grupos excluidos y discriminados siendo que la Organización Mundial de la Salud entiende por inequidades las desigualdades evitables en materia de salud entre grupos de población en un mismo país o entre países.

El crecimiento de los ingresoses una medida parcial y errónea sobre el desarrollo de los países. El postulado del PNUD sobre que la riqueza de una nación lo constituye su gente se rescata en la creación de la medida del Índice de Desarrollo Humano (IDH). El IDH es un índice compuesto del índice de salud, índice de educación y el índice de ingresos.En México, con ayuda de este indicador se puede mostrar el avance del país en términos sociales, pero asimismo se muestran las enormes brechas al interior del mismo.

En este trabajo se muestra aprecia que los grandes problemas de México es la situación de pobreza que tiene su población, la vulnerabilidad por carencias sociales, carencias por acceso a los servicios de salud, carencias por acceso a la seguridad social y por tener ingresos inferiores a la línea de bienestar. Si ordenamos todas estas carencias se tendría que las peores situadas lo son igualmente en todos y cada uno de estos rubros, por lo que se puede concluir que las regiones más pobres y con mayor desigualdad son asimismo las regiones que más carencias tienen y a las que se les da peor atención por parte del Estado.

\section{BIBLIOGRAFIA}

CONAPO (2010). Índice de marginación por localidad. CONAPO, 2010, México.

BANCO MUNDIAL. Banco Mundial en línea: http://datos.bancomundial.org/tema/salud.

GATTINI, C Y RUÍZ, P. (2012). "Salud en Sudamérica - Panorama de la situación y de las políticas y sistemas de salud", Edición de 2012, OPS/OMS, Washington, 2012.

INFORME DE DESARROLLO HUMANO (1990), Programa de las Naciones Unidas para el desarrollo, PNUD, Nueva York, Estados Unidos. 
INFORME DE DESARROLLO HUMANO (2011), Programa de las Naciones Unidas para el desarrollo, PNUD, Nueva York, Estados Unidos.

ORGANIZACIÓN MUNDIAL DE LA SALUD(2012). Estadísticas sanitarias mundiales 2012. OMS, Ginebra

PNUD (2012), "El índice de desarrollo humano en México: cambios metodológicos e información para las entidades federativas", sitio web:

http://www.undp.org.mx/IMG/pdf/Boletin_IDH.pdf, consultado el 23 de octubre de 2012. México.

SEN, A., (1986), « The standard of living", Cambridge University, sitio web: http://www.tannerlectures.utah.edu/lectures/documents/sen86.pdf, consultado el 20 de noviembre de 2011

SEN, A., 1992, "Nuevo examen de la desigualdad", Alianza editorial, Madrid, España.

SEN, A. (1998), "Capital humano y capacidad humana", Cuadernos de Economía, v. XVII, Núm. 29, pp.67-72, Colombia. Sitio web:

http://www.revistas.unal.edu.co/index.php/ceconomia/article/view/11496, consultado el 9 de diciembre de 2011.

SEN, A. (1998-a), "El futuro del estado de bienestar", conferencia pronunciada en el "Círculo de economía" de Barcelona, España.

SEN, A., (1993), "La vida y la muerte como indicadores económicos", Investigación y ciencia, 202:6-13, sitio web:

http://www.galeon.com/gentealternativa/tribunaoradores/tribuna801.htm, consultado el 25 de noviembre de 2011.

RAWLS, J., (1973), Justicia Distributiva, traducción del capítulo IV de Economic Justice, Penguin Books, Inc, 1973, Capítulo $4^{\circ}$, Sección 13, pp 319-362. Sitio web: www.cepchile.cl/dms/archivo_792_1057/rev24_rawls.pdf, consultado el 01 de diciembre de 2011.

RAWLS, J. (1995), “Teoría de la justicia”, FCE, 2 edición, México. 